\title{
FGF-23 in fibrous dysplasia of bone and its relationship to renal phosphate wasting
}

\author{
Mara Riminucci, ${ }^{1,2}$ Michael T. Collins, ${ }^{3}$ Neal S. Fedarko, ${ }^{4}$ Natasha Cherman, ${ }^{3}$ \\ Alessandro Corsi, ${ }^{1,5}$ Kenneth E. White, ${ }^{6}$ Steven Waguespack, ${ }^{7}$ Anurag Gupta, ${ }^{3}$ \\ Tamara Hannon, ${ }^{8}$ Michael J. Econs, ${ }^{6,9}$ Paolo Bianco, ${ }^{2,3,5}$ and Pamela Gehron Robey ${ }^{3}$ \\ ${ }^{1}$ Dipartimento di Medicina Sperimentale, Università dell'Aquila, L'Aquila, Italy \\ ${ }^{2}$ Parco Scientifico Biomedico San Raffaele, Rome, Italy \\ ${ }^{3}$ Craniofacial and Skeletal Diseases Branch, National Institute of Dental and Craniofacial Research, \\ National Institutes of Health, Department of Health and Human Services, Bethesda, Maryland, USA \\ ${ }^{4}$ Division of Geriatrics, Department of Medicine, Johns Hopkins University, Baltimore, Maryland, USA \\ ${ }^{5}$ Dipartimento di Medicina Sperimentale e Patologia, Università "La Sapienza," Rome, Italy \\ ${ }^{6}$ Department of Medicine, Indiana University School of Medicine, Indianapolis, Indiana, USA \\ ${ }^{7}$ Department of Endocrine Neoplasia and Hormonal Disorders, University of Texas, MD Anderson Cancer Center, \\ Houston, Texas, USA \\ ${ }^{8}$ Department of Pediatrics and \\ ${ }^{9}$ Department of Medical and Molecular Genetics, Indiana University School of Medicine, Indianapolis, Indiana, USA
}

\begin{abstract}
FGF-23, a novel member of the FGF family, is the product of the gene mutated in autosomal dominant hypophosphatemic rickets (ADHR). FGF-23 has been proposed as a circulating factor causing renal phosphate wasting not only in ADHR (as a result of inadequate degradation), but also in tumor-induced osteomalacia (as a result of excess synthesis by tumor cells). Renal phosphate wasting occurs in approximately $50 \%$ of patients with McCune-Albright syndrome (MAS) and fibrous dysplasia of bone (FD), which result from postzygotic mutations of the GNAS1 gene. We found that FGF-23 is produced by normal and FD osteoprogenitors and bone-forming cells in vivo and in vitro. In situ hybridization analysis of FGF-23 mRNA expression identified "fibrous" cells, osteogenic cells, and cells associated with microvascular walls as specific cellular sources of FGF23 in FD. Serum levels of FGF-23 were increased in FD/MAS patients compared with normal agematched controls and significantly higher in FD/MAS patients with renal phosphate wasting compared with those without, and correlated with disease burden bone turnover markers commonly used to assess disease activity. Production of FGF-23 by FD tissue may play an important role in the renal phosphate-wasting syndrome associated with FD/MAS.
\end{abstract}

J. Clin. Invest. 112:683-692 (2003). doi:10.1172/JCI200318399.

Received for publication March 19, 2003, and accepted in revised form June 17, 2003

Address correspondence to: Paolo Bianco, Dipartmento di Medicina Sperimentale e Patologia, Università "La Sapienza," Viale Regina Elena 324, Rome 00161, Italy.

Phone: 39-06-444-1049; Fax: 39-06-494-0896;

E-mail: p.bianco@flashnet.it.

Conflict of interest: I.E. White and M.J. Econs are the inventors of FGF-23, which Indiana University has licensed to Kirin Pharmaceuticals.

Nonstandard abbreviations used: autosomal dominant hypophosphatemic rickets (ADHR); X-linked hypophosphatemic rickets (XLH); tumor-induced osteomalacia (TIO); matrix extracellular phosphoglycoprotein (MEPE); secreted frizzled-related protein-4 (sFRP-4); fibrous dysplasia (FD); stimulatory $\mathrm{G}$ protein $\left(\mathrm{G}_{\mathrm{s}}\right)$; McCune-Albright syndrome (MAS); Institutional Review Board (IRB); maximum rate of reabsorption of phosphate relative to the GFR (TmP/GFR); genomic DNA (gDNA); protein nucleic acid (PNA); poly-methyl methacrylate (MMA); human trabecular bone (HTB): non-phosphate wasters (NPW); phosphate wasters (PW); alkaline phosphatase (ALP); osteonectin (ON); osteocalcin (OC);

bone sialoprotein (BSP).

\section{Introduction}

Phosphate-wasting disorders include heritable autosomal dominant hypophosphatemic rickets (ADHR); $\mathrm{X}$-linked hypophosphatemic rickets (XLH), and acquired tumor-induced osteomalacia (TIO), conditions manifesting impaired renal reabsorption of phosphate, hypophosphatemia, and skeletal mineralization defects (1). Missense mutations in the FGF-23 gene, encoding a novel member of the FGF family, are the cause of ADHR (2). Inadequate endopeptidase cleavage of the mutated protein may result in increased FGF-23 serum levels and bioactivity $(3,4)$. On the other hand, FGF-23 is produced by most tumors associated with TIO, a rare paraneoplastic form of phosphaturic, hypophosphatemic osteomalacia (5-9). Although other candidate factors have been identified, including matrix extracellular phosphoglycoprotein (MEPE) (10) and secreted frizzled-related protein-4 (sFRP-4) (11), circulating FGF-23 represents one candidate "phosphatonin", the longpostulated (and long-sought) putative humoral fac- 
tor that would regulate renal phosphate handling and be secreted in excess by TIO-causing tumors ( 8 , $12,13)$. A unifying picture of human phosphaturic disorders would emerge from these recent observations. Both inactivating mutations of PHEX, a neutral endopeptidase gene (in X-linked hypophosphatemia) $(14,15)$, and missense mutations in FGF-23 itself (in ADHR) (2), may result in impaired FGF-23 degradation $(3,4)$. Inappropriate production of FGF-23 by phosphaturic tumors, on the other hand, may cause TIO. FGF-23 would represent the crossroad of inherited and acquired metabolic derangements, finding in hypophosphatemia, renal phosphate wasting, and skeletal mineralization defects, a final common pathway (13).

Fibrous dysplasia (FD), a genetic, noninherited disease caused by somatic activating missense mutations of GNAS1 (16) (encoding the $\alpha$ subunit of the stimulatory $\mathrm{G}$ protein, $\mathrm{G}_{\mathrm{s}}$ ), is a non-neoplastic disease affecting the skeleton either in isolation (monostotic and polyostotic forms), or in variable combinations with endocrine and cutaneous abnormalities (McCune-Albright syndrome, MAS) (17-20). Hypophosphatemic rickets/osteomalacia is recognized as a rare complication of FD/MAS (21-23). Less-pronounced degrees of renal phosphate wasting are detected in approximately $50 \%$ of $\mathrm{FD} /$ MAS patients (24) and are commonly associated with localized mineralization defects in FD bone $(25,26)$, which contribute significantly to disease morbidity (27). We thus hypothesized that FGF-23 might be associated with the phosphate-wasting disorder observed in FD/MAS patients and sought to determine (i) if FGF-23 is expressed and synthesized in FD tissue and cells (ii) whether circulating FGF-23 could be detected in FD/MAS patients at levels higher than normal subjects, and (iii) whether there is a relationship between FGF-23 and phosphate wasting in FD/MAS patients. Using a combination of RT-PCR, in situ hybridization, ELISA of media conditioned by normal and FD marrow stromal cells and trabecular bone cells, and measurements of FGF-23 in sera, we show here that, indeed, FGF-23 is expressed in FD tissue and osteogenic cells and that high levels of circulating FGF-23 correlate with renal phosphate wasting in $\mathrm{FD} / \mathrm{MAS}$ patients.

\section{Methods}

Patients and samples. Forty-nine FD/MAS patients (age range 4-58 years) were enrolled in Institutional Review Board-approved (IRB-approved) studies of FD/MAS at the National Institutes of Health and gave written informed consent for the studies. Diagnosis of FD/MAS was confirmed based on clinical history, radiographic findings, histopathological findings, and mutation analysis of the GNAS1 gene. Due to known effects of bisphosphonate treatment on markers of bone turnover, patients receiving bisphosphonates were not included in this cohort. Sera and urine specimens were collected while patients were inpatients and on a hospital diet consisting of the following nutrients: calcium $(850 \mathrm{mg}$; range 690-1,066 mg), phosphorus (1,312 $\mathrm{mg}$; range $1,065-1,744 \mathrm{mg}$ ); potassium (3,596 $\mathrm{mg}$; range $3,107-4,595 \mathrm{mg})$, and sodium $(5,111 \mathrm{mg}$; range $4,282-6,412 \mathrm{mg})$. Sera and urine specimens from all 49 patients were used for FGF-23, phosphate, and maximum rate of reabsorption of phosphate relative to the GFR (TmP/GFR) determinations. Sera from 283 normal control subjects (age range 1-84 years) were collected under an IRB-approved protocol at Indiana University. Biopsy specimens from the FDinvolved iliac crest from five patients (two nonphosphaturic and three phosphaturic) were used for in situ hybridization analysis as detailed below. Normal controls included one iliac crest specimen taken from the uninvolved bone of a FD patient, two normal iliac crest samples from non-FD patients, and one sample of fracture callus. Cell cultures were established from FD tissue of five patients (three nonphosphaturic and two phosphaturic) and normal bone of three agematched normal controls as detailed below.

GNAS1 mutation analysis. Mutations were determined in genomic DNA (gDNA) isolated from either tissue or cells using the standard protocol of the DNeasy Tissue Kit (QIAGEN Inc., Valencia, California, USA). Purified gDNA (200-500 ng) was amplified using either standard primers for direct DNA sequencing or using a protein nucleic acidbased (PNA-based) primer, which blocks amplification of the normal allele. Amplification for direct sequencing used $1 \mu \mathrm{g}$ of the forward primer: $5^{\prime}$ TGACTATGTGCCGAGCGA-3' (exon 7), and the reverse primer: 5'-AACCATGATCTCTGTTATATAA-3' (intron G) (GenBank Accession no. M21142.1, bases 272-289 and 521-542, respectively) in a $100-\mu$ reaction. Samples were heated to $94^{\circ} \mathrm{C}$ for 15 minutes, then cycled 35 times at $94^{\circ} \mathrm{C}$ for 60 seconds, $55^{\circ} \mathrm{C}$ for 30 seconds, and $72^{\circ} \mathrm{C}$ for 60 seconds, and terminated for 7 minutes at $72^{\circ} \mathrm{C}$. To block amplification of the wild-type allele, a PNA-based primer that blocks amplification of the normal allele was used as reported previously (25). PCR products were purified using the QiAmp PCR purification kit (QIAGEN Inc.) and sequenced with a dRhodamine dyeterminator cycle sequencing with Ampli Taq and the Perkin-Elmer Applied Biosystems 377 automated sequencer (Palo Alto, California, USA).

In situ bybridization. A 755-bp sequence within the human FGF-23 cloned into the pcDNA3.1(+) plasmid (8) was inserted into pBluescript plasmid vector (Invitrogen Life Technologies, Carlsbad, California, USA) and used as template for transcription of digoxigenin-labeled RNA probes. The fragment, excised with EcoRI and BamHI restriction enzymes (New England Biolabs Inc., Beverly, Massachusetts, USA), was purified by $0.8 \%$ agarose gel and a QIAquik Gel Extraction Kit (QIAGEN Inc.), and then subcloned 
into pBluescript vector. RNA probes were synthesized using the DIG RNA Labeling Kit (Boehringer Mannheim GmbH, Mannheim, Germany), according to the manufacturer's instructions. BamHI and T7 RNA polymerase and EcoRI and T3 RNA polymerase were used to synthesize the antisense probe and sense probes, respectively. Limited alkaline hydrolysis was used to generate fragments of both probes.

All biopsy specimens were fixed in freshly prepared phosphate-buffered $4 \%$ formaldehyde for 24 hours. After washing in PBS, samples were decalcified in buffered EDTA and paraffin embedded. Additional specimens were embedded in poly-methyl methacrylate (MMA), as described previously (26), and undecalcified sections obtained from MMA blocks were stained with von Kossa stain. Deparaffinized sections were digested with $20 \mu \mathrm{g} / \mathrm{ml}$ proteinase $\mathrm{K}$ (Invitrogen Life Technologies) in $10 \mathrm{mM}$ Tris-HCl, $1 \mathrm{mM}$ EDTA, $\mathrm{pH} 8.0$, at $37^{\circ} \mathrm{C}$ for 20 minutes, washed in $0.1 \mathrm{M}$ glycine (Invitrogen Life Technologies), then PBS, and preincubated with mRNA in situ hybridization solution (DAKO Corp., Carpinteria, California, USA) at $50^{\circ} \mathrm{C}$ for 2 hours. Sense and anti-sense RNA probes were diluted in hybridization solution at a final concentration of 5 $\mathrm{ng} / \mu \mathrm{l}$. After hybridization at $55^{\circ} \mathrm{C}$ overnight, sections were washed as follows: $4 \times$ SSC (four times for 5 minutes), $2 \times$ SSC (two times for 5 minutes), $1 \times$ SSC (once for 10 minutes), $0.5 \times \mathrm{SSC}$ (once for 10 minutes) at room temperature, and $0.2 \times$ SSC plus $0.2 \%$ BSA (Sigma-Aldrich, St. Louis, Missouri, USA) twice for 20 minutes at $60^{\circ} \mathrm{C}$. Hybridization was detected using alkaline phosphatase-conjugated antidigoxygenin (Boehringer Mannheim $\mathrm{GmbH}$ ) with $p$-nitroblue tetrazolium chloride and 5-bromo-4-chloro-3-indolyl-phosphate $p$-toluidine salt (Boehringer Mannheim GmbH), as described previously (28).

Immunohistochemistry. Deparaffinized sections were incubated for immunohistochemistry using Ab's against alkaline phosphatase (LF-47), osteonectin (BON-1), osteopontin (LF-123), osteocalcin (LF-32), and bone sialoprotein (LF-83), kindly provided by Larry W. Fisher (National Committee for Clinical Laboratory Studies, NIH, Department of Health and Human Services, Bethesda, Maryland, USA) (29). All antisera were used in an indirect immunoperoxidase protocol as described previously (30).

Isolation of human bone marrow stromal cells. Marrow stromal cell cultures were established as described previously (31). Briefly, cells were released by scraping bone marrow into nutrient medium consisting of $\alpha$-MEM (Biofluids Inc., Rockville, Maryland, USA), $20 \%$ FBS (Life Technologies Inc., Gaithersburg, Maryland, USA), penicillin, streptomycin, and glutamine (Biofluids Inc.). Single cell suspensions were plated in $75-\mathrm{mm}^{2}$ flasks $\left(0.02 \times 10^{5}-1.7 \times 10^{5}\right.$ nucleated cells/ $\mathrm{cm}^{2}$ ) to generate multicolony-derived strains (by passaging all colonies together at day 10) or in Petri dishes $\left(10^{4}\right.$ nucleated cells $\left./ 100 \mathrm{~mm}^{2}\right)$ to generate single colony-derived strains. The medium used was the same as above, which supports proliferation rather than differentiation of stromal progenitors, as described previously (32). After 10-14 days, individual colonies were isolated using cloning cylinders and trypsin release and expanded in cell number (32). After mutation analysis of gDNA by direct DNA sequencing, individual clonal lines were identified as normal or mutant. Multiple mutant clonal strains from each sample were combined to derive $100 \%$ mutant strains. Cell strains at the third passage were used for RT-PCR and ELISA analysis of FGF-23 production.

Isolation of human trabecular bone cells. Human trabecular bone (HTB) cells (osteoblast-like cells) derived from normal and FD bone specimens were established in culture under IRB-approved procedures as described previously (33). Briefly, fragments of trabecular bone were minced to the consistency of sand, washed extensively, and treated with bacterial collagenase $(250 \mathrm{U} / \mathrm{ml}$ collagenase $\mathrm{P}$; Boehringer Mannheim $\mathrm{GmbH}$ ) to remove all soft tissue and associated cells. The collagenase-treated fragments were placed in low-calcium $(0.02 \mathrm{mM})$ nutrient medium containing a 50:50 mixture of DMEM/Ham's F-12 K with $10 \%$ FBS, glutamine, penicillin, and streptomycin. Cells grew from the fragments starting at 1-2 weeks and were passaged after reaching confluence at approximately 4 weeks. Cells were plated in the same medium, but with normal levels of calcium $(1.8 \mathrm{mM})$, for analysis of FGF-23 expression. These cells have been shown previously to have all characteristics of osteoblast-like cells (33).

$R T-P C R$. Total RNA was isolated from both fresh FD tissue and cell strains using TRIzol (Sigma-Aldrich), and cDNA was generated using random primers and the SuperScript First-Strand Synthesis System (Invitrogen Life Technologies), both according to the manufacturers' instructions. The target cDNA sequence was amplified using two different primer sets. In the first set the forward primer was $5^{\prime}$-GTGCAGCGTCTGCAGCATG-3' (bases 185-203), and the reverse primer was 5'-CCATGCAGAGGTATCTTCTG-3' (bases 416-435). In the second set, the forward primer was $5^{\prime}$-CACTGCCATCACATACTAAC-3' (bases 2308-2327), and the reverse primer was 5'-ATACTGCCACATGACGAG-3' (bases 2663-2680) (GenBank Accession no. 15055547). Standard PCR reactions were performed using reagents from the Perkin Elmer Kit and 2.5 units of Platinum Taq polymerase. After $94^{\circ} \mathrm{C}$ for 5 minutes, the reaction was run for 30 cycles at $94^{\circ} \mathrm{C}, 55^{\circ} \mathrm{C}$, and $72^{\circ} \mathrm{C}$ for 45 seconds at each temperature, with a final extension at $72^{\circ} \mathrm{C}$ for 5 minutes, which generated a 251-bp amplification product from the first primer set, and $94^{\circ} \mathrm{C}, 49^{\circ} \mathrm{C}, 72^{\circ} \mathrm{C}$ for 45 seconds at each temperature, with final extension at $72^{\circ} \mathrm{C}$ for 5 minutes, which generated a 373-bp amplification product from the second primer set. All amplification products were verified by DNA sequencing.

ELISA analysis. Conditioned medium was generated by near-confluent cultures of normal and mutant 
stromal cells and HTB cells maintained in serum-free $\alpha$-MEM. Media samples were collected at 24 hours and frozen at $-80^{\circ} \mathrm{C}$ until analysis by ELISA, using a commercially available two-site ELISA for the FGF-23 $\mathrm{C}$ terminus according to the manufacturer's instructions (Immutopics International, San Clemente, California, USA). In additional experiments, normal stromal cells and normal human osteoblast-like cells were treated with serum-free medium containing 1 mM dibutyryl cAMP (Sigma-Aldrich) (28), and medium was collected 24 hours later.

Serum measurements. Sera from 49 FD patients (21 pediatric patients, younger than 18 years old; 28 adult patients, older than 18 years old) were assayed for FGF-23 activity using a commercially available two-site ELISA for the FGF-23 C terminus, according to the manufacturer's instructions (Immutopics International). As a control, FGF-23 was also measured in sera of normal pediatric (younger than 18 years, $n=79$ ) and adult donors (older than 18 years, $n=204$ ). Statistical analysis was performed using ANOVA and the Scheffe F test; regression analysis was used to determine potential correlations between serum levels of FGF-23 and other metabolic parameters.

TmP/GFR and serum phosphate determination. Renal phosphate handling was assessed by calculating the TmP/GFR using serum samples obtained after an overnight fast and urine samples from 24-hour urine collections, using an adaptation of the technique and nomogram of Walton and Bijovet $(24,34,35)$. Patients were considered to have normal excretion of phosphate in the urine (non-phosphate wasters, NPW) or renal phosphate wasting (phosphate wasters, PW) if their value of $\mathrm{TmP} / \mathrm{GFR}$ was higher or lower, respectively, than the lower limit of the ageand gender-specific normal range (36). Twenty-fourhour urine samples were used to determine $\mathrm{TmP} /$ GFR after having determined that calculation of $\mathrm{TmP} / \mathrm{GFR}$ using fasting second-morning voided

\section{Figure 1}

In situ hybridization analysis of FGF-23 expression in FD of bone. Biopsy specimens of FD-involved iliac crest. Serial sections from two FD phosphaturic patients were hybridized with antisense and sense RNA probes for FGF-23 mRNA. (a-f) Patient 1. (a and b) Expression of FGF-23 is clearly observed both in the fibrous component of the lesion and in bone cells associated with abnormal bone trabeculae (a). No signal is observed in sections hybridized with the sense (control) probe (b). (c and $\mathbf{d}$ ) Strong expression of FGF-23 mRNA is observed in the abnormal bone cells associated with the surface of abnormal bone trabeculae (double arrows) and with individual osteocytes therein (arrowheads) (d). (e and $\mathbf{f}$ ) Expression of FGF-23 mRNA is also observed in cells (endothelial cells and/or pericytes) within the walls of microvessels in the FD tissue (single arrows). (g-i) Patient 2. Strong hybridization signal in abnormal bone cells ( $\mathbf{g}, \mathbf{i}, \mathbf{j}$; thick arrows in $\mathbf{j}$ ) and in spindle-shaped fibroblastic cells in the fibrous tissue (thin arrows in $\mathbf{j}$ ). No signal is detected in sections hybridized with the sense probe (h). $f t$, fibrous tissue; $b$, bone; $b v$, blood vessel lumen. urine specimens and aliquots from 24-hour urine samples generates virtually identical values consistently on the same side of the lower limit of the range for each patient. Serum phosphate was determined for each patient and compared in the groups of adult and pediatric NPW and PW patients.

Assessment of disease burden. To test whether phosphate wasting and FGF-23 concentrations are correlated to disease burden, we assessed disease burden in all patients, using a validated tool that calculates the percentage of the skeleton involved with FD (Chen, C., et al., manuscript submitted for publication). This technique takes advantage of the fact the FD is avid for ${ }^{99} \mathrm{Tc}-\mathrm{MDP}$. All patients underwent ${ }^{99} \mathrm{Tc}-\mathrm{MDP}$ bone scintigraphy, and the scans were assessed as to the relative amount of each segment of the skeleton that incorporated the isotope. These segments were then normalized relative to the percentage of the total skeleton that each segment represents. These values were then summed, and a value was generated that represents the amount of the skeleton involved with FD, that is, disease burden. In addition, conventional markers of bone turnover, commonly taken as indices of disease extent and activity in FD $(27,37)$, were also assessed by standard commercially available techniques.
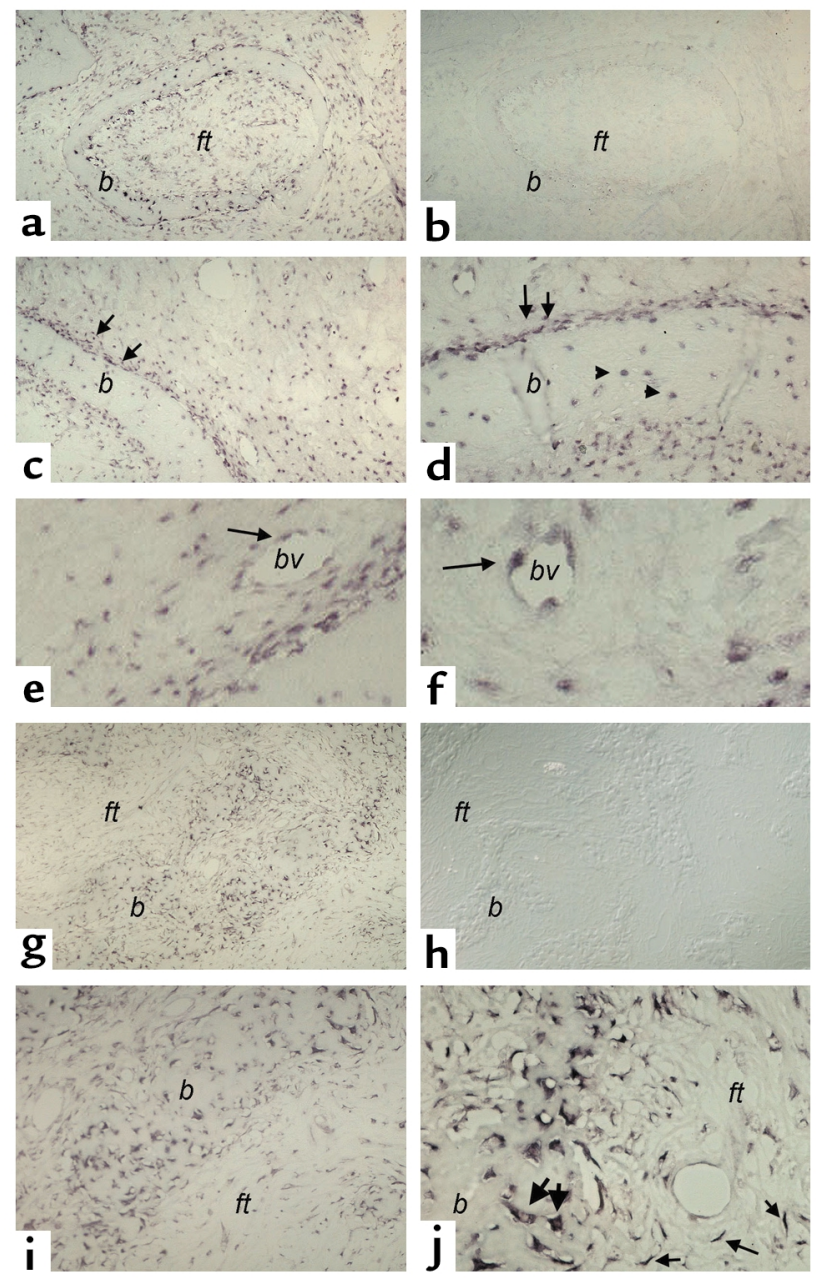

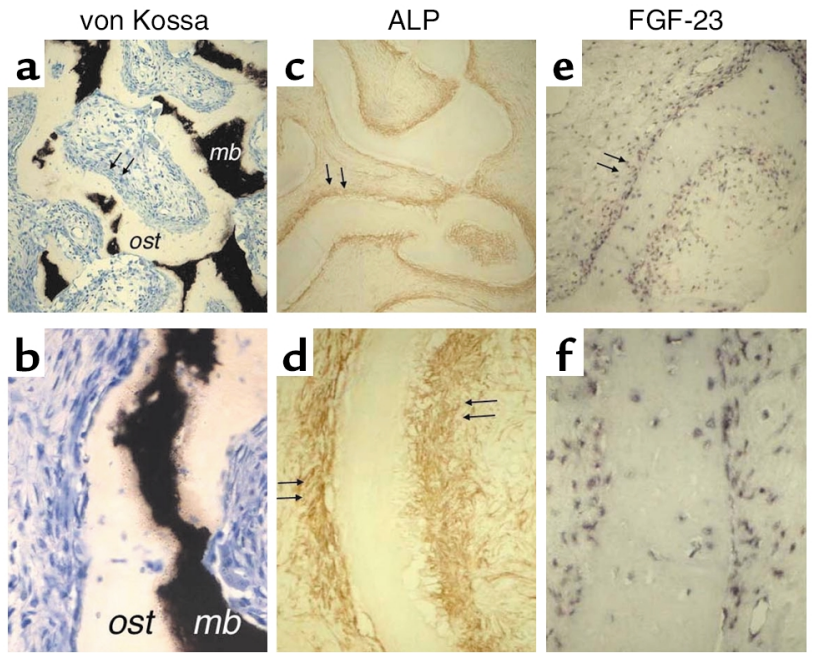

\section{Figure 2}

Immunohistochemical characterization of FGF-23-producing cells in FD. Undecalcified MMA sections ( $\mathbf{a}$ and $\mathbf{b}$ ) and paraffin sections (c-f) were prepared from the same tissue sample. (a and $\mathbf{b}$ ) Von Kossa staining of MMA sections demonstrates the severe mineralization defect in FD bone, reflected in a significant excess of osteoid (ost) and paucity of mineralized bone matrix $(\mathrm{mb}$; black with von Kossa). (c and d) Immunolocalization of ALP. Cells layered onto trabecular bone surfaces (double arrows in $\mathbf{c}$ and $\mathbf{d}$ ) are intensely ALP positive. (e and $\mathbf{f}$ ) FGF-23 in situ hybridization. The codistribution of the hybridization signal with the immunoreactivity for ALP (double arrows in $\mathbf{c}$ and $\mathbf{e}$, respectively) is shown. Also note that osteocytes within FD bone do express FGF-23 (f), but are ALP negative $(\mathbf{d})$.

\section{Results}

FGF-23 is expressed in FD tissue. FD lesions are essentially composed of a "fibrous" component, previously shown to consist of the accumulation of immature osteogenic cells and abnormal bone trabeculae associated with abnormal osteoblasts and osteocytes. In situ hybridization analysis of tissue from phosphaturic patients revealed strong and specific hybridization signal for FGF-23 mRNA, both in the fibroblastic cells within the fibrous tissue and in the abnormal osteoblasts and osteocytes associated with the dysplastic bone trabeculae (Figure 1, a, c, d, g, i, j). Although hybridization signal was also observed in cells associated with microvascular walls within the FD lesions (Figure 1, e and f), the fibroblastic cells and cells physically associated with FD bone trabeculae obviously represented the major component of FD tissue in which FGF-23 mRNA was detected. The osteogenic nature of these cells was confirmed by immunohistochemical characterization (Figures 2 and 3). FGF-23positive fibroblastic (stromal) cells within FD tissue also expressed the early markers of osteogenic differentiation, alkaline phosphatase (ALP), and osteonectin $(\mathrm{ON})$ (Figure 2, c and d; Figure 3, b and c), but these fibroblastic cells did not express the late markers of osteogenic differentiation, osteocalcin (OC) (not shown) and bone sialoprotein (BSP) (Figure 3d), consistent with previous reports (28). Mature osteogenic cells associated with FD bone trabeculae (FD osteoblasts and FD osteocytes) that expressed FGF-23 mRNA were also characteristically positive for ALP (osteoblasts) and ON (osteoblasts and some osteocytes) (Figure 2, c and d; Figure 3, b and c) and variably positive for OC (osteoblasts and recently formed osteocytes) (Figure 3e). The immunohistochemical findings are summarized in Table 1. Since a state of phosphate wasting is not an obligate feature of FD, FGF-23 mRNA expression was also investigated in FD tissue obtained from nonphosphaturic patients (Figure 4). In situ hybridization analysis revealed essentially similar patterns of expression in both instances.

FGF-23 is expressed in normal bone. To investigate whether the expression of FGF-23 in FD osteogenic cells was specifically related to the abnormal nature of the FD tissue, or rather to the osteogenic nature of the lesional cells, we analyzed the expression of FGF-23 in normal human bone. In adult trabecular bone, in which most bone surfaces are not involved in active bone formation events, a weak but specific signal was observed in osteocytes and flattened bone-lining cells (inactive osteoblasts; Figure 5, a and c). In sections of
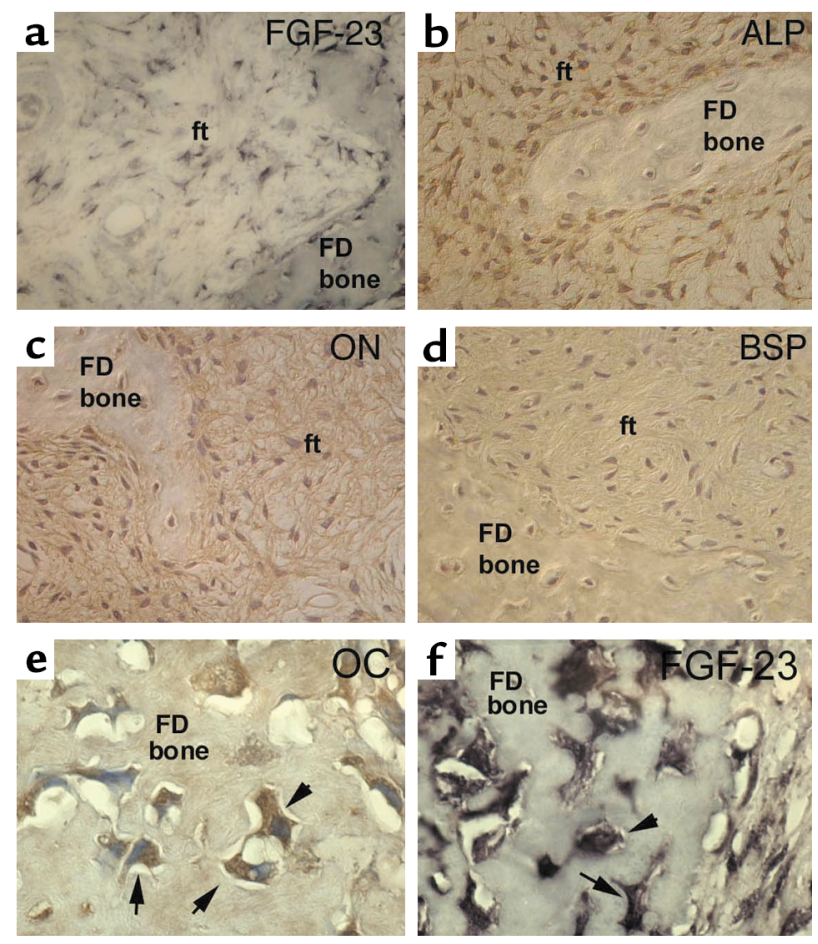

Figure 3

Immunohistochemical characterization of FGF-23-producing cells in FD. Stromal cells in the fibrous tissue ( $\mathrm{ft}$ ) express FGF-23 mRNA (a), are intensely positive for the early markers of osteogenic differentiation, ALP (b) and ON (c), but are negative for the markers of late osteogenic differentiation, BSP (d) and OC (not shown). Both ALP and ON immunostaining result in decoration of a dense network of delicate cell processes of FD stromal cells. Mature osteogenic cells embedded as osteocytes in newly formed FD bone express the late marker of osteogenic differentiation, and OC (not shown) (e), and express FGF-23 mRNA (f). 
Table 1

Characterization of FGF-23-expressing cells in FD sections

\begin{tabular}{|c|c|c|c|c|c|}
\hline & $\begin{array}{c}\text { FD } \\
\text { stromal cells }\end{array}$ & $\begin{array}{c}\text { FD } \\
\text { osteoblasts }\end{array}$ & $\begin{array}{c}\text { FD } \\
\text { osteocytes }\end{array}$ & $\begin{array}{c}\text { FD } \\
\text { osteoclasts }\end{array}$ & $\begin{array}{c}\text { Endothelia/ } \\
\text { pericytes }\end{array}$ \\
\hline ALP & ++ & ++ & - & - & $+/-$ \\
\hline ON & ++ & ++ & $+/-$ & - & $+/-$ \\
\hline OPN & - & $+/-$ & - & + & - \\
\hline BSP & $-/+*$ & $-/+^{*}$ & $-/+^{*}$ & - & - \\
\hline OC & - & $+/-$ & $+/-$ & - & - \\
\hline FGF-23 & ++ & ++ & ++ & $+/-$ & ++ \\
\hline
\end{tabular}

OPN, osteopontin; ++, consistently positive, high staining intensity; +/-, positive in some cells, weak to moderate staining intensity; $-/_{+*}^{*}$, negative with polyclonal antiserum, positive in some cells with a monoclonal $\mathrm{Ab} ;-$, negative.

fracture callus, where intense bone formation activity takes place, all bone surfaces were lined by active osteoblasts in which a strong hybridization signal was consistently observed (Figure 5e). Newly formed osteocytes (Figure $5 \mathrm{~g}$ ) and periosteal osteoprogenitor cells (Figure 5i) were also intensely labeled. No signal was observed in control sections hybridized with the sense probe. From these studies we concluded that FGF-23 mRNA is expressed in osteogenic cells within $\mathrm{FD}$, regardless of the occurrence of a phosphate-wasting state, and is also expressed in osteogenic cells in normal human bone, particularly during phases of active bone remodeling.

FGF-23 is produced by GNAS1-mutated and normal osteogenic cells. RT-PCR analysis of RNA extracted from fresh FD tissue confirmed the expression of FGF-23 mRNA (Figure 6a). Stromal cells (which include osteoprogenitor cells) grown from FD tissue also expressed FGF-23 mRNA by RT-PCR. Since cultures of FDderived stromal cells usually include variable proportions of mutated and nonmutated cells (31), expression of FGF-23 mRNA was also analyzed in pure strains of GNAS1-mutated stromal cells obtained by cell cloning, and levels were similar to those expressed by nonclonal FD cells. This confirmed that FGF-23 is, in fact, expressed by osteogenic cells carrying the causative mutation of FD (Figure 6a). Expression of FGF-23 mRNA in culture, however, was not restricted to GNAS1-mutated cells, since it was also observed in stromal cells derived from the bone marrow of normal donors. FGF-23 mRNA was also expressed by osteoblast-like cells (HTB cells) derived from normal and FD bone (Figure 6a). The identity of the amplified cDNA sequence was verified by direct sequencing of the amplification product in all cases.

The actual production of FGF-23 protein by normal and FD-derived stromal cells and human osteoblastlike cells was confirmed by analyzing conditioned media by ELISA. Measurable levels of FGF-23 were observed in media conditioned by either normal or FDderived cell cultures for 24 hours (Figure 6b) and were not detected in either serum-free medium or in serumcontaining medium not exposed to cells. No significant difference was observed between normal and FD cells or between FD cells from nonphosphaturic and phosphaturic patients. In addition, when normal stromal cells or osteoblast-like cells were treated with dibutyryl-cAMP to mimic the effects of GNAS1 mutations (28), there was no increase in FGF-23 secretion (data not shown). In keeping with observations made in situ, in vitro studies indicated that expression of FGF- 23 by osteogenic FD cells is not a specific phenotypic effect of the disease genotype and were mirrored by similar expression in normal osteogenic cells. Also in keeping with the results of in situ studies, in vitro data revealed expression of FGF-23 both in immature (stromal progenitor cells) and more mature osteogenic cells (trabecular bone-derived osteoblast-like cells).

Serum levels of FGF-23 are elevated in FD patients. To determine whether production of FGF-23 within FD tissue would be reflected into higher levels of circulating FGF-23 in FD patients, we measured FGF-23 in the serum of 49 patients with FD and 283 normal controls. Levels of circulating FGF-23 were significantly higher in patients with FD as compared with normal agematched controls (Table 2).

Serum levels of FGF-23 correlate with renal phosphate wasting in FD patients. To investigate the potential impact of circulating FGF-23 on renal phosphate wasting, FD patients of pediatric or adult age range were further subdivided into NPW and PW groups. Each individual patient was assigned to either group based on determination of TmP/GFR (lower or higher than the lower
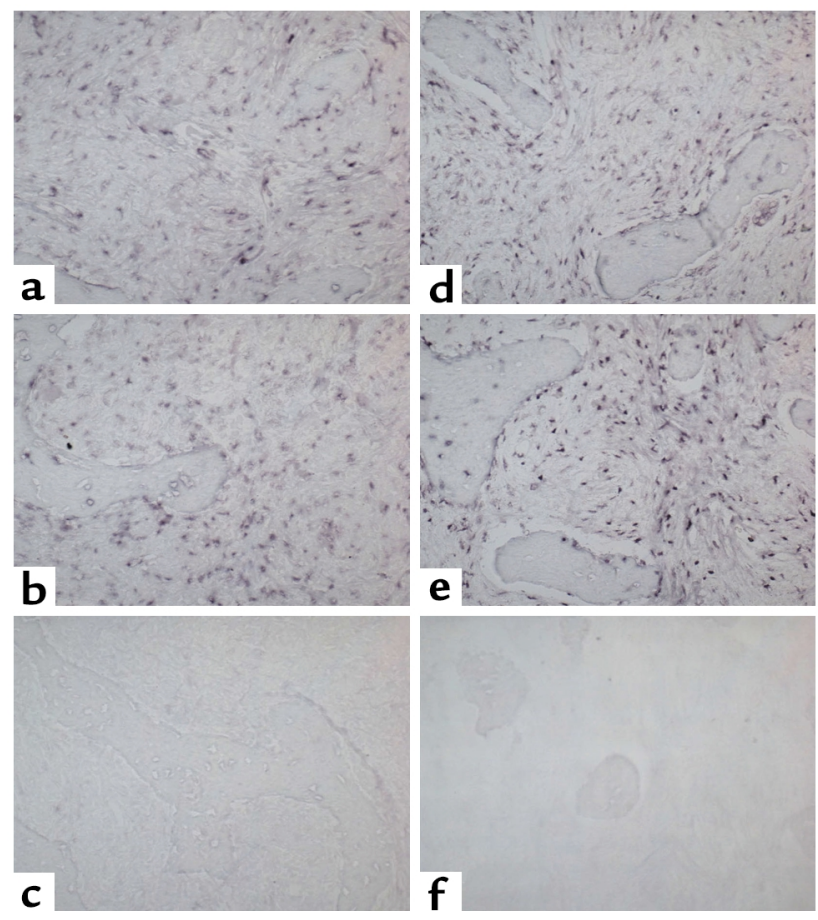

Figure 4

Expression of FGF-23 in FD tissue from patients with normal phosphatemia and TmP/GFR. (a-c) Images are from one patient. (d-f) Images from a second patient. ( $c$ and $\mathbf{f}$ ) Sections hybridized with the sense (control) probe. Signal for FGF-23 mRNA is observed in both cases ( $\mathbf{a}$ and $\mathbf{b}, \mathbf{d}$ and $\mathbf{e}$ ). 

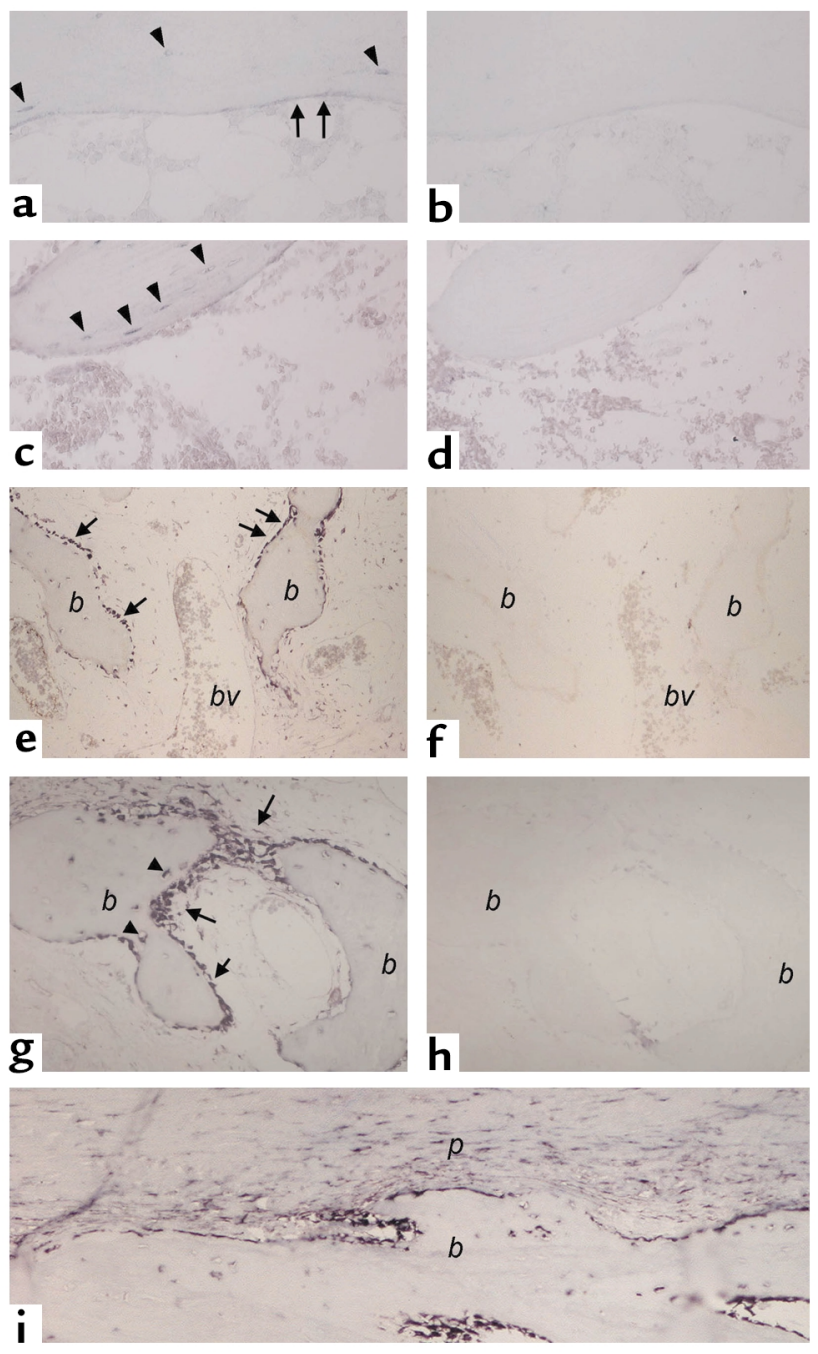

limit of the normal range for the specific age and gender, respectively). The mean serum phosphate was significantly lower in the PW groups compared with NPW groups (pediatric, $3.19 \pm 0.37 \mathrm{mg} / \mathrm{dl}$ versus $4.89 \pm 0.47$ $\mathrm{mg} / \mathrm{dl}, P=0.001$, ANOVA; adult, $2.88 \pm 0.33 \mathrm{mg} / \mathrm{dl}$ versus $3.69 \pm 0.38 \mathrm{mg} / \mathrm{dl}, P=0.0001$, ANOVA). Levels of FGF-23 in both PW and NPW patients were then analyzed in comparison with each other and to agematched controls. Significantly higher levels of serum FGF-23 were observed in PW patients compared with either normal controls or NPW patients in the same age range. In contrast, no statistically significant difference was observed between serum FGF-23 levels of NPW patients and those of age-matched controls (Tables 2 and 3). Regression analysis revealed a highly significant negative correlation of serum FGF-23 levels with serum phosphate levels and TmP/GFR, both in pediatric and adult patients (Figure 7).

Serum levels of FGF-23 correlate with disease burden in FD patients. Since FGF-23 was found to be expressed both in $\mathrm{FD}$ and in normal bone and to be produced at similar levels by mutated and nonmutated osteogenic cells, we hypothesized that the apparent heterogeneity of

\section{Figure 5}

In situ hybridization analysis of FGF-23 expression in normal bone. (a-d) Normal trabecular bone (iliac crest). (a and c) Antisense probe; (b and $\mathbf{d}$ ) sense (control) probe. Hybridization signal is observed over flattened bone-lining cells (surface-associated quiescent osteogenic cells [a, arrows] and osteocytes [a and $\mathbf{c}$, arrowheads]). (e-i) Normal fracture callus. $b v$, blood vessel lumen. $(\mathbf{e}, \mathbf{g}, \mathbf{i})$ Antisense probe. ( $\mathbf{f}$ and $\mathbf{h}$ ) Sense (control) probe. Intense hybridization signal is observed in osteoblasts (e and $\mathbf{g}$, arrows) actively involved in deposition of reparative bone $(b)$ and in newly formed osteocytes therein (g, arrowheads). (i) Periosteum and subperiosteal bone. Note the expression of FGF-23 in periosteal osteoprogenitor cells $(p)$.

serum levels of FGF-23 in FD patients, reflected in the nonobligatory occurrence of renal phosphate wasting and hypophosphatemia, could essentially reflect disease burden. To test this hypothesis, we evaluated the extent of skeletal involvement in PW and NPW patients using a bone scintigraphy-based technique. A significantly higher disease burden was observed in PW patients compared with NPW (pediatric, $36.42 \pm 15.28$ versus $15.38 \pm 10.69, P=0.0049$, ANOVA; adult, $35.77 \pm 22.91$ versus. $15.01 \pm 18.39, P=0.005$, ANOVA), and linear regression analysis revealed that a significant positive correlation between disease burden and serum FGF-23 could be demonstrated for both pediatric and adult FD patients (Figure 8, a and b). Likewise, linear regression analysis revealed a significant correlation between serum FGF-23 levels with serum ALP and urinary deoxypyridinoline, commonly used as indicators of disease extent and activity in FD $(27,37)$ (Figure 8, c, d, e, f). Tight correlations were also observed between the extent of skeletal involvement and other markers of bone turnover (data not shown).

\section{Discussion}

The quest for circulating phosphaturic factor(s) has made a major advance with the recognition that an FGF family member, FGF-23, is encoded by the gene mutated in ADHR (2) and made resistant to endopeptidase degradation by the missense mutations underlying the disease $(3,4)$. The detection of high levels of FGF-23 mRNA and protein in most TIO-causing tumors and of FGF-23 in the serum of patients with

\section{Table 2}

FGF-23 in serum of normal controls and patients with FD

\begin{tabular}{lcccc}
\hline Age range & Groups & $n$ & FGF-23 Mean \pm SD & $P$ value \\
All & Normal & 283 & $63.2 \pm 44.9$ & \\
$(1-84$ years $)$ & FD & 49 & $128.2 \pm 101.8$ & 0.000 \\
& NPW & 25 & $76.9 \pm 66.4$ & \\
& PW & 24 & $181.6 \pm 105.9$ & \\
Pediatric & Normal & 78 & $64.2 \pm 34.4$ & \\
(<18 years) & FD & 21 & $175.7 \pm 120.84$ & 0.0019 \\
& NPW & 12 & $112.3 \pm 78.1$ & \\
& PW & 9 & $260.3 \pm 118.5$ & \\
Adult & Normal & 205 & $62.8 \pm 48.52$ & 0.0077 \\
$(>18$ years $)$ & FD & 28 & $92.6 \pm 67.42$ & \\
& NPW & 13 & $44.4 \pm 28.3$ & \\
& PW & 15 & $134.4 \pm 63.4$ & \\
\hline
\end{tabular}



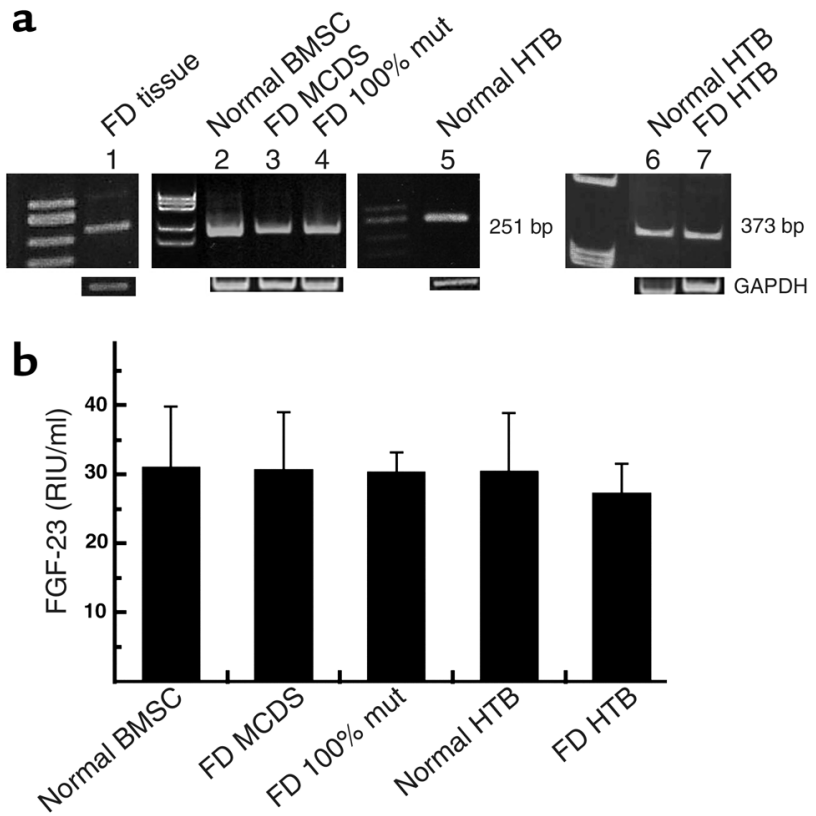

TIO $(8,12,38)$ suggests that FGF- 23 may be seen as a circulating phosphaturic factor of potentially broader significance. While additional factors, such as MEPE (10) or secreted FRP-4 (11), have been proposed as potential regulators of renal phosphate handling, strong experimental evidence has been provided in animal models for the potential role of FGF-23 as a phosphaturic factor (12). A phosphate-wasting syndrome resembling TIO (24) and localized mineralization defects $(25,26)$ occur in a substantial proportion of patients with FD. A clear-cut picture of generalized, overt hypophosphatemic osteomalacia is also observed in rare cases of this disorder (21-23). We therefore analyzed the tissue source, serum levels, and effect on renal phosphate handling of FGF-23 in FD.

We have demonstrated that FGF-23 mRNA is expressed in FD tissue, predominantly by cells of osteogenic lineage comprising the so-called fibrosis characteristic of the lesion and the abnormal osteoblasts and osteocytes associated with the dysplastic bone trabeculae. Production of FGF-23, by lesional tissue and by cells derived from it, is reflected by serum levels that are significantly higher in FD patients than in normal controls and correlate inversely with phosphatemia and renal phosphate reabsorption in FD patients. These data support the notion that FGF-23 may act as a phosphaturic factor in FD and may explain the occurrence of localized (lesional) or generalized mineralization defects in the skeleton of some FD patients.

Our studies, however, also reveal aspects of the relationships between FGF-23 and phosphate metabolism that are unique to $\mathrm{FD}$ and in turn highlight novel facets of the pathophysiology of FGF-23 and phosphate metabolism at large. We have shown that FGF-23 mRNA is expressed in normal bone cells, and it is produced in culture by FD and normal bone-derived cells, as well as by wild-type and GNAS1-mutated cells, at apparently

\section{Figure 6}

Expression of FGF-23 mRNA and protein. (a) RT-PCR primer sets that generate 251 - and 373 -bp products. FGF-23 mRNA was identified in fresh FD tissue (lane 1), in normal, nonclonal bone marrow stromal cells (BMSC) and multicolony-derived strains (MCDS) from a normal donor (lane 2), nonclonal strains of FD-derived stromal cells (comprising mutated and nonmutated cells) (lane 3 ), in pure strains of GNAS1-mutated stromal cells (FD 100\% mut) (lane 4), and in HTB cells from normal (lanes 5 and 6) and FD (lane 7) donors. The identity of the amplification product was verified by DNA sequencing. (b) Conditioned medium from serum-free cultures of normal bone marrow stromal cells (BMSC), FD-derived nonclonal multicolony (mixed) strains and pure strains of GNAS1-mutated stromal cells (FD 100\% mut), and normal and FD HTB cells (HTB) were harvested at 24 hours and analyzed by ELISA for production ofFGF-23. Measurable levels of FGF-23 were observed in all cultures, with no significant difference between GNAS1-mutation status or type of cell culture. The values represent the combined results of two separate experiments and error bars represent standard error of the mean. Standard medium (containing 15\% FBS) does not contain detectable FGF-23. RIU, relative immunoreactive unit.

similar levels. These data indicate that skeletal cells represent a previously unrecognized physiological source of FGF-23 and that production of FGF-23 by FD cells cannot be accounted for as a consequence of the GNAS1 mutations, but rather reflect their osteogenic nature (28). Likewise, the detection of abnormally high levels of serum FGF-23 in FD patients and the occurrence of a renal phosphate-wasting syndrome cannot be explained solely by the development of a clone of GNAS1-mutated cells in the body, which would produce inappropriate levels of FGF-23. This is in keeping with the observation that renal phosphate wasting is not an obligate occurrence in FD patients, all of whom harbor some mutated FD osteogenic cells in their skeletal lesion(s), but rather occurs in only approximately 50\% of FD patients. As we have shown, patients with renal phosphate wasting are characterized by a high disease burden. On the other hand, serum levels of FGF-23 are not significantly elevated in patients with FD who are free of a phosphate-wasting syndrome. These patients, as we have shown, are characterized by a significantly lower disease burden. Thus, clinical observations are

\section{Table 3}

Comparisons of serum FGF-23 levels in normal donors and FD patients without and with phosphate wasting

\begin{tabular}{lcc}
\hline Age range & Comparisons (ANOVA) & Scheffe F test \\
All & Normal versus NPW & 2.78 \\
(1-84 years) & Normal versus PW & $27.76^{\mathrm{A}}$ \\
& NPW versus PW & $28.30^{\mathrm{A}}$ \\
Pediatric & Normal versus NPW & 1.74 \\
(<18 yrs) & Normal versus PW & $18.86^{\mathrm{A}}$ \\
& NPW versus PW & $15.35^{\mathrm{A}}$ \\
Adult & Normal versus NPW & 0.876 \\
$(>18$ yrs $)$ & Normal versus PW & $14.32^{\mathrm{A}}$ \\
& NPW versus PW & $23.25^{\mathrm{A}}$
\end{tabular}

ASignificant at greater than or equal to $95 \%$. 


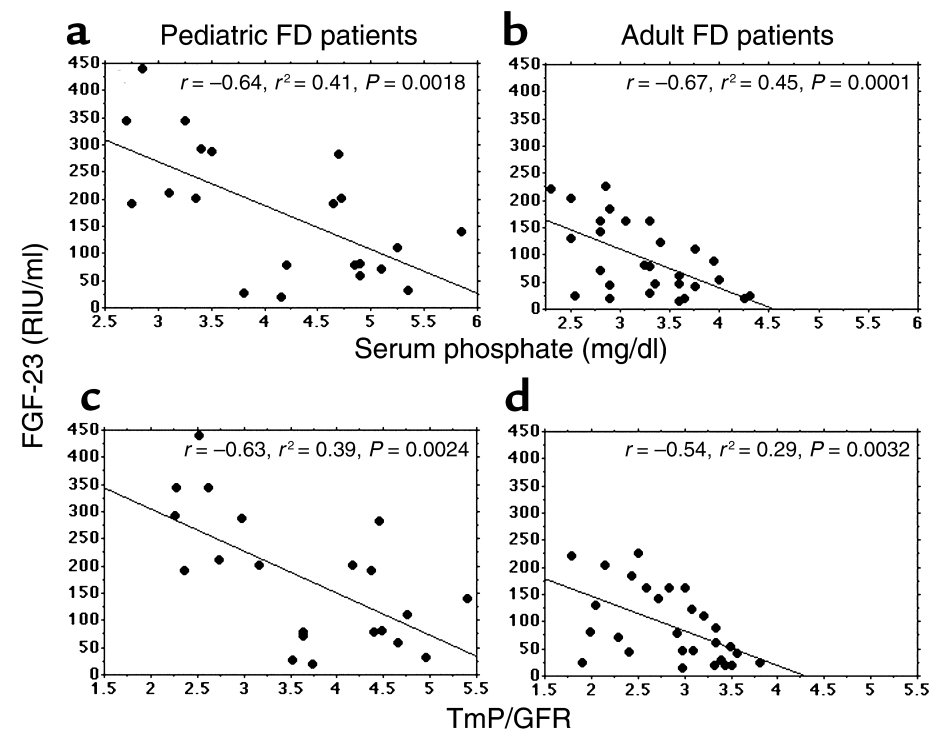

Figure 7

Negative correlation of serum FGF-23 levels with serum phosphate ( $\mathbf{a}$ and $\mathbf{b}$ ) and TmP/GFR ( $\mathbf{c}$ and $\mathbf{d}$ ) in pediatric and adult FD patients.

marked differences in the local regulation of expression and metabolism of FGF-23 in FD (cells) versus phosphaturic tumor (cells), which are, as yet, not well understood. In the general scheme of phosphaturic disorders associated with excess bioactive FGF-23, however, FD represents a novel scenario, somewhat distinct from the one represented by TIO. In TIO, excess levels of serum FGF-23 are generated by an abnormally high release of the factor at the single cell level (inappropriate cellular production). In $\mathrm{FD}$, an excess of osteogenic cells, normally competent to release FGF-23, accumulate in fibrous dys-

easily reconciled with, and in fact explained by, the lack of major differences between normal and FD cells when FGF-23 production is evaluated at the single cell level. In addition, the observation that disease burden significantly correlates with serum FGF-23 and serum phosphate and urinary deoxypyridinoline levels in FD patients fits well with the observed incidence of phosphate wasting in patients with more extensive disease.

Current views of the role of FGF-23 in phosphate metabolism inscribe both inherited and acquired phosphaturic disorders into a common paradigm (39). Excess circulating FGF-23 is postulated to represent a final common pathway of $\mathrm{XLH}, \mathrm{ADHR}$, and TIO. In XLH and ADHR, the excess bioactive FGF-23 would result from lack of adequate degradation, either due to lack of a putative degrading enzyme activity (in XLH) or to gain-of-function mutations resulting in reduced degradability of FGF-23 itself (in ADHR). In this scheme, TIO would represent a situation of inappropriate, and possibly ectopic, production of excess FGF-23 by the so-called phosphaturic tumors. By comparing the serum levels of FGF-23 observed in FD patients in our study with those recently reported for patients with TIO (38), it is notable that several kilograms of FD tissue (as reflected by disease burden) are required to generate serum levels of FGF-23 equivalent to that which is generated by tumors that often do not exceed the size of $1 \mathrm{~cm}$ and are mostly benign. This implies

\section{Figure 8}

Positive correlation of serum FGF-23 with disease burden assessed as percentage of skeletal involvement in bone scintigrams ( $\mathbf{a}$ and $\mathbf{b}$ ) and with markers often used to assess FD activity, ALP ( $\mathbf{c}$ and $\mathbf{d}$ ) and deoxypyridinoline (e and $\mathbf{f}$ ), for pediatric and adult FD patients. CR, creatinine. plastic tissues (28) (inappropriate amount of cells normally producing FGF-23).

In summary, our results provide direct evidence for the production of FGF-23 by normal, active osteogenic cells, and by osteogenic cells in fibrous dysplastic lesions, for elevated levels of circulating FGF-23 in FD patients and for their relationship to an associated state of renal phosphate wasting and hypophosphatemia. These data provide a novel insight into mechanisms underlying the more (hypophosphatemic rickets; refs. 21-23) and less
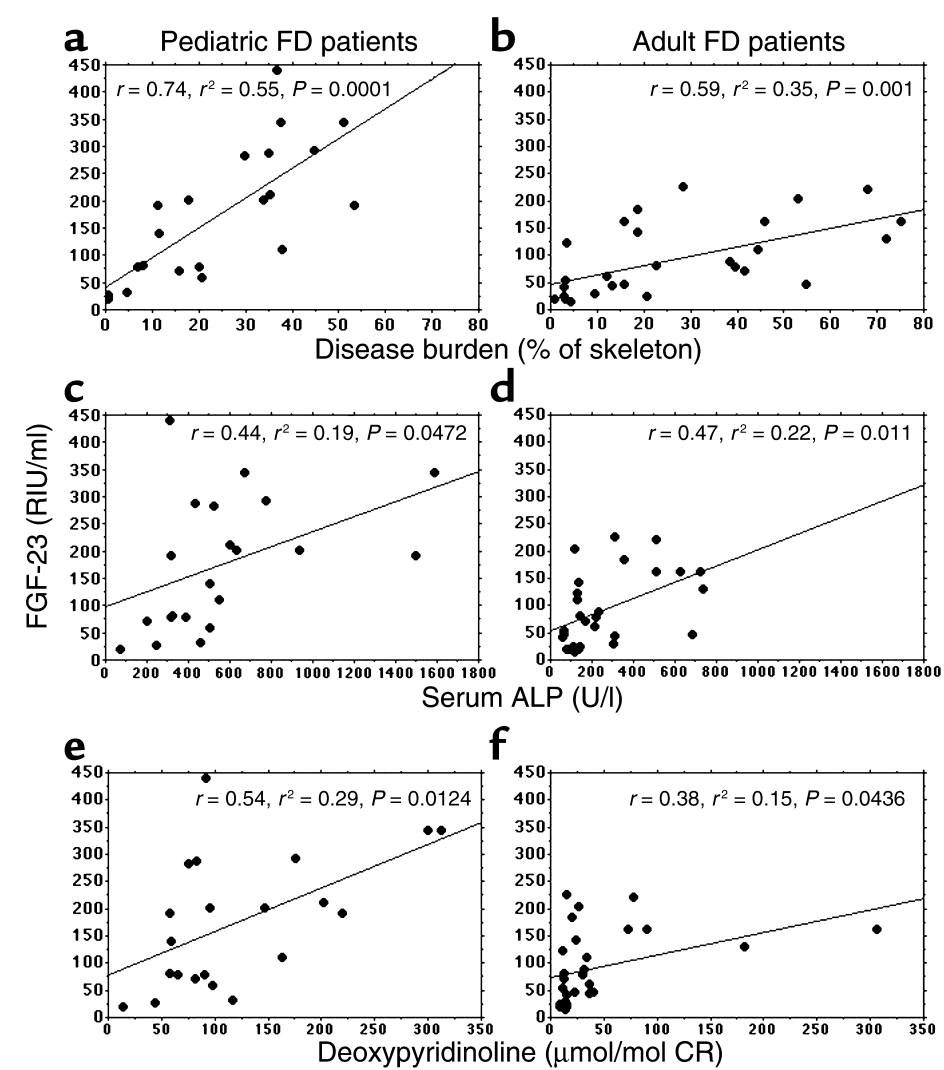
(localized intralesional osteomalacia; ref. 25) conspicuous consequences of renal phosphate wasting in FD that are increasingly recognized as an important determinant of skeletal disease severity $(26,27)$. In addition, they extend the range of human disorders for which FGF-23 is clinically and biologically relevant as a candidate for a circulating phosphaturic factor and the range of pathophysiological mechanisms leading to excess circulating bioactive FGF-23. Finally, they raise the interesting possibility that bone cells themselves may act as an endocrine organ regulating systemic phosphate metabolism.

\section{Acknowledgments}

The support of Telethon Fondazione Onlus Grant E1029 (to P. Bianco) is gratefully acknowledged, as well as $\mathrm{NIH}$ grants DK-063934 (to K.E. White) and AR-42228, AG-18397, AR-02095, and AR-47866 (to M.J. Econs).

1. DiMeglio, L.A., White, K.E., and Econs, M.J. 2000. Disorders of phosphate metabolism. Endocrinol. Metab. Clin. North Am. 29:591-609.

2. The ADHR Consortium. 2000. Autosomal dominant hypophosphataemic rickets is associated with mutations in FGF-23. The ADHR Consortium. Nat. Genet. 26:345-348.

3. White, K., et al. 2001. Autosomal-dominant hypophosphatemic rickets (ADHR) mutations stabilize FGF-23. Kidney Int. 60:2079-2086.

4. Shimada, T., et al. 2002. Mutant FGF-23 responsible for autosomal dominant hypophosphatemic rickets is resistant to proteolytic cleavage and causes hypophosphatemia in vivo. Endocrinology. 143:3179-3182.

5. Weidner, N. 1991. Review and update: oncogenic osteomalacia-rickets. Ultrastruct. Pathol. 15:317-333.

6. Wilkins, G.E., et al. 1995. Oncogenic osteomalacia: evidence for a humoral phosphaturic factor. J. Clin. Endocrinol. Metab. 80:1628-1634.

7. Kumar, R. 2000. Tumor-induced osteomalacia and the regulation of phosphate homeostasis. Bone. 27:333-338.

8. White, K.E., et al. 2001. The autosomal dominant hypophosphatemic rickets (ADHR) gene is a secreted polypeptide overexpressed by tumors that cause phosphate wasting. J. Clin. Endocrinol. Metab. 86:497-500.

9. Fukumoto, S., and Yamashita, T. 2002. Fibroblast growth factor-23 is the phosphaturic factor in tumor-induced osteomalacia and may be phosphatonin. Curr. Opin. Nephrol. Hypertens. 11:385-389.

10. Rowe, P.S., et al. 2000. MEPE, a new gene expressed in bone marrow and tumors causing osteomalacia. Genomics. 67:54-68.

11. De Beur, S.M., et al. 2002. Tumors associated with oncogenic osteomalacia express genes important in bone and mineral metabolism. J. Bone Miner. Res. 17:1102-1110.

12. Shimada, T., et al. 2001. Cloning and characterization of FGF-23 as a causative factor of tumor-induced osteomalacia. Proc. Natl. Acad. Sci. U. S. A. 98:6500-6505.

13. Strewler, G.J. 2001. FGF23, hypophosphatemia, and rickets: has phosphatonin been found? Proc. Natl. Acad. Sci. U. S. A. 98:5945-5946.

14. Dixon, P.H., et al. 1998. Mutational analysis of PHEX gene in X-linked hypophosphatemia. J. Clin. Endocrinol. Metab. 83:3615-3623.

15. The HYP Consortium. 1995. A gene (PEX) with homologies to endopeptidases is mutated in patients with X-linked hypophosphatemic rickets. The HYP Consortium. Nat. Genet. 11:130-136.

16. Weinstein, L.S., et al. 1991. Activating mutations of the stimulatory G protein in the McCune-Albright syndrome. N. Engl. J. Med. 325:1688-1695.
17. Albright, F., Butler, A.M., Hampton, A.O., and Smith, P. 1937. Syndrome characterized by osteitis fibrosa disseminata, areas of pigmentation and endocrine dysfunction with precocious puberty in females. N. Engl. J. Med. 216:727-746.

18. McCune, D.J., and Bruch, H. 1937. Progress in pediatrics: osteodystrophia fibrosa. Am. J. Dis. Child. 54:806-848.

19. Lichtenstein, L. 1938. Polyostotic fibrous dysplasia. Arch. Surg. 36:874-879.

20. Lichtenstein, L., and Jaffe, H.L. 1942. Fibrous dysplasia of bone: a condition affecting one, several or many bones, the graver cases of which may present abnormal pigmentation of skin, premature sexual development, hyperthyroidism and still other extraskeletal abnormalities. Arch. Pathol. 33:777-797.

21. Ryan, W.G., Nibbe, A.F., Schwartz, T.B., and Ray, R.D. 1968. Fibrous dysplasia of bone with vitamin D resistant rickets: a case study. Metabolism. 17:988-998.

22. Dent, C.E., and Gertner, J.M. 1976. Hypophosphataemic osteomalacia in fibrous dysplasia. Q. J. Med. 45:411-420.

23. Dachille, R.D., Goldberg, J.S., Wexler, I.D., and Shons, A.R. 1990. Fibrous dysplasia-induced hypocalcemia/rickets. J. Oral Maxillofac. Surg. 48:1319-1322.

24. Collins, M.T., et al. 2001. Renal phosphate wasting in fibrous dysplasia of bone is part of a generalized renal tubular dysfunction similar to that seen in tumor-induced osteomalacia. J. Bone Miner. Res. 16:806-813.

25. Bianco, P., et al. 2000. Mutations of the GNAS1 gene, stromal cell dysfunction, and osteomalacic changes in non-McCune-Albright fibrous dysplasia of bone. J. Bone Miner. Res. 15:120-128.

26. Corsi, A., et al. 2003. Osteomalacic and hyperparathyroid changes in fibrous dysplasia of bone: core biopsy studies and clinical correlations. J. Bone Miner. Res. 18:1235-1246.

27. Bianco, P., Robey, P.G., and Wientroub, S. 2003. Fibrous dysplasia of bone. In Pediatric Bone - biology and disease. F.H. Glorieux, J. Pettifor, and H. Juppner, editors. Academic Press. New York, New York, USA. 509-539.

28. Riminucci, M., et al. 1997. Fibrous dysplasia of bone in the McCuneAlbright syndrome: abnormalities in bone formation. Am. J. Pathol. 151:1587-1600.

29. Fisher, L.W., Stubbs, J.T., and Young, M.F. 1995. Antisera and cDNA probes to human and certain animal model bone matrix noncollagenous proteins. Acta Orthop. Scand. Suppl. 266:61-65.

30. Hsu, S.M., Raine, L., and Fanger, H. 1981. Use of avidin-biotin-peroxidase complex $(\mathrm{ABC})$ in immunoperoxidase techniques: a comparison between $\mathrm{ABC}$ and unlabeled antibody (PAP) procedures. J. Histochem. Cytochem. 29:577-580.

31. Bianco, P., et al. 1998. Reproduction of human fibrous dysplasia of bone in immunocompromised mice by transplanted mosaics of normal and Gsalpha-mutated skeletal progenitor cells. J. Clin. Invest. 101:1737-1744.

32. Kuznetsov, S.A., et al. 1997. Single-colony derived strains of human marrow stromal fibroblasts form bone after transplantation in vivo. J. Bone Miner. Res. 12:1335-1347.

33. Robey, P.G., and Termine, J.D. 1985. Human bone cells in vitro. Calcif. Tissue Int. 37:453-460.

34. Bijvoet, O.L., Morgan, D.B., and Fourman, P. 1969. The assessment of phosphate reabsorption. Clin. Chim. Acta. 26:15-24.

35. Kenny, A.P., and Glen, A.C. 1973. Tests of phosphate reabsorption. Lancet. 2:158.

36. Payne, R.B. 1998. Renal tubular reabsorption of phosphate (TmP/GFR): indications and interpretation. Ann. Clin. Biochem. 35:201-206.

37. Chapurlat, R., and Meunier, P.J. 2000. Fibrous dysplasia of bone. Bailliere's Clin. Rheumatol. 14:385-398.

38. Jonsson, K.B., et al. 2003. Fibroblast growth factor 23 in oncogenic osteomalacia and X-linked hypophosphatemia. N. Engl. J. Med. 348:1656-1663.

39. Carpenter, T.O. 2003. Oncogenic osteomalacia - a complex dance of factors. N. Engl. J. Med. 348:1705-1708. 